\title{
New Avenues for Phosphodiesterase Inhibitors in Asthma
}

This article was published in the following Dove Press journal:

Journal of Experimental Pharmacology

\author{
Maria Gabriella Matera' \\ Josuel Ora $\mathbb{D}^{2}$ \\ Francesco Cavalli ${ }^{2}$ \\ Paola Rogliani (iD) ${ }^{2,3}$ \\ Mario Cazzola iD ${ }^{3}$ \\ 'Department of Experimental Medicine, \\ University of Campania "Luigi Vanvitelli", \\ Naples, Italy; ${ }^{2}$ Respiratory Diseases Unit, \\ "Tor Vergata" University Hospital, Rome, \\ Italy; ${ }^{3}$ Department of Experimental \\ Medicine, University of Rome "Tor \\ Vergata", Rome, Italy
}

Introduction: Phosphodiesterases (PDEs) are isoenzymes ubiquitously expressed in the lungs where they catalyse cyclic adenosine monophosphate (cAMP) and cyclic guanosine monophosphate (GMP), which are fundamental second messengers in asthma, thereby regulating the intracellular concentrations of these cyclic nucleotides, their signaling pathways and, consequently, myriad biological responses. The superfamily of PDEs is composed of 11 families with a distinct substrate specificity, molecular structure and subcellular localization. Experimental studies indicate a possible role in asthma mainly for PDE3, PDE4, PDE5 and PDE7. Consequently, drugs that inhibit PDEs may offer novel therapeutic options for the treatment of this disease.

Areas Covered: In this article, we describe the progress made in recent years regarding the possibility of using PDE inhibitors in the treatment of asthma.

Expert Opinion: Many data indicate the potential benefits of PDE inhibitors as an add-on treatment especially in severe asthma due to their bronchodilator and/or anti-inflammatory activity, but no compound has yet reached the market as asthma treatment mainly because of their limited tolerability. Therefore, there is a growing interest in developing new PDE inhibitors with an improved safety profile. In particular, the research is focused on the development of drugs capable of interacting simultaneously with different PDEs, or to be administered by inhalation. CHF 6001 and RPL554 are the only molecules that currently are under clinical development but there are several new agents with interesting pharmacological profiles. It will be stimulating to assess the impact of such agents on individual treatable traits in specially designed studies.

Keywords: asthma, phosphodiesterases, phosphodiesterase inhibitors, bifunctional drugs, treatable traits therapeutic approach

\section{Introduction}

For several years, theophylline, which is an orally active non-selective phosphodiesterase (PDE) inhibitor with other relevant pharmacologic activities that are likely to contribute to its efficacy, such as inhibition of phosphoinositide 3 -kinase- $\delta$, adenosine receptor antagonism and increased activity of certain histone deacetylases that deacetylate lysine residues in chromatin, thereby silencing gene transcription, ${ }^{1}$ has been used in the management of asthma because of its bronchodilatory and anti-inflammatory effects. ${ }^{2}$ However, at least in part, theophylline has fallen out of favor because of its adverse side-effect profile, and this has led to the search for more effective and safer PDE inhibitors. ${ }^{3}$

In a valuable review published in 1991 on phosphodiesterase (PDE) inhibitors examined as new opportunities for the treatment of asthma, Torphy and Undem so closed their article
Correspondence: Mario Cazzola Dipartimento di Medicina Sperimentale, Università di Roma Tor Vergata, Rome, Italy

Email mario.cazzola@uniroma2.it
Journal of Experimental Pharmacology 2021:13 29|-302 
After having been moribund for many years, interest in phosphodiesterase inhibitors as agents for asthma has undergone a striking resurgence, particularly with respect to the potential therapeutic advantages of isozyme selective phosphodiesterase inhibitors. ${ }^{4}$

However, 30 years later, roflumilast is the only PDE inhibitor to enter the market and its use has exclusively been approved for the treatment of chronic obstructive pulmonary disease (COPD). ${ }^{5}$ This contrasts with the opinion repeatedly expressed by many researchers and clinicians that PDE inhibitors could be useful in the treatment of asthma because of their bronchodilator and/or antiinflammatory activities. ${ }^{6-10}$

The predominant opinion is that the unsuccessful development of PDE inhibitors for the treatment of asthma is at least partly due to the many clinical failures in this area and the side effect profile of all the tested drugs. ${ }^{11}$ Nonetheless, at least some researchers believe that PDE inhibitors with a tolerable safety profile could be beneficial as an add-on treatment in severe asthma because they have many different mechanisms of action in the lungs. ${ }^{8-10}$ In fact, adding roflumilast to ICS provided additional $\mathrm{FEV}_{1}$ improvement from baseline to 24 weeks. ${ }^{12}$ There is experimental evidence that roflumilast stimulates both glucocorticoid receptor $\alpha(\mathrm{GR} \alpha)$ mRNA synthesis and GR $\alpha$ 's transcriptional activity in bronchial epithelial cells and enhances dexamethasone's ability to suppress pro-inflammatory mediator production, in a GR $\alpha$ dependent manner. ${ }^{13}$ Furthermore, significant interest has been expressed in using PDE inhibitors in the group of patients who have overlapping characteristics of asthma and COPD. ${ }^{14,15}$

In this review, we discuss the reason why selective PDE inhibitors are of interest for therapeutic application in asthma treatment and emphasize the possibility to combine various PDE inhibitors to increase their therapeutic effectiveness.

\section{Search Strategy and Selection Criteria}

The literature search was conducted in MEDLINE and Embase databases via Ovid, to identify articles published until January 30, 2021, using the following search terms: PDE, PDE inhibitors, asthma. The search was expanded also to clinicaltrial.gov, clinicaltrialsregister.eu, and jmacct.med.or.jp. Moreover, the name of each compound was independently searched on PubMed to avoid missing data.

\section{PDE Families and Asthma}

Human genome contains 21 PDE genes, which are divided into PDE families (PDE1 to PDE11) and each family contains different subtypes. ${ }^{16}$ PDEs catalyse the hydrolysis of cyclic adenosine monophosphate (cAMP) and cyclic guanosine monophosphate (GMP), thereby regulating the intracellular concentrations of these cyclic nucleotides, their signaling pathways and, consequently, myriad biological responses in health and disease. ${ }^{16}$ As we have understood by now, different PDE isozymes selectively regulate cAMP or cGMP signaling in different subcellular microdomains, and individual PDEs are likely involved in specific locations at certain time-points based on different stimulations/ activations. ${ }^{17,18}$ There are more than 100 isoforms of PDEs identified, each of them exerts distinctive, non-overlapping and non-redundant physiological effects on the human body, which means that there exists a myriad of unique PDE isozymes that can be targeted to evoke multitude of beneficial biological responses. ${ }^{19}$ Therefore, rather than focusing on a single PDE, we must consider the participation of multiple PDE variants in a complex signaling network involving central regulatory mechanisms. ${ }^{20}$

In any case, PDE4, PDE7, and PDE8 are cAMPspecific PDEs, PDE5, PDE6, and PDE9 are cGMPspecific PDEs, whereas PDE1, PDE2, PDE3, PDE10 and PDE11 hydrolyse both cAMP and cGMP. ${ }^{19}$

PDEs are expressed in different lung cell types such as airway and vascular smooth muscle cells, epithelial cells, and fibroblasts and in inflammatory and immune cells (eosinophils, neutrophils, monocytes, macrophages, T-lymphocytes and B-lymphocytes). ${ }^{18}$

cAMP is an important second messenger in asthma because it is immunomodulatory virtually for all cell types involved in its pathogenesis and regulates the airway smooth muscle (ASM) tone via the $\beta_{2}$-adrenergic $\left(\beta_{2}\right.$-AR)soluble adenylyl cyclase (sAC)-cAMP signaling pathway. ${ }^{21}$ When its levels rise, cAMP causes relaxation of the ASM and inhibits numerous immune and inflammatory responses that are fundamental in the pathophysiology of asthma, including activation and proliferation of $\mathrm{T}$ lymphocytes, production of superoxide anion in eosinophils, and chemotaxis of eosinophils provoked by inflammatory mediators. ${ }^{22}$ Furthermore, increased cAMP levels can affect mucociliary clearance directly through the activation and acceleration of ciliary motility, which depends on the balance between $\mathrm{Ca}^{2+}$ ions and cAMP, and indirectly by affecting allergic or other 
inflammation. ${ }^{23}$ This is important in asthma because ciliary dysfunction is a feature of moderate to severe asthma. ${ }^{24}$

Also cGMP is an important second messenger in asthma because it regulates the vascular smooth muscle relaxation via the nitric oxide (NO)-soluble guanylyl cyclase (sGC)-cGMP pathway ${ }^{25}$ and is also involved in evoking bronchodilation in human small airways as is activating the $\beta_{2}$-AR-sAC-cAMP pathway. ${ }^{26}$ Recently, it has been observed that a majority of human ASM cells derived from severe asthma donors express an $\mathrm{sGC}$ that is primarily or completely unresponsive toward $\mathrm{NO}$, making them dysfunctional for NO-sGC-cGMP signaling. ${ }^{27}$

Transcriptomic data demonstrate the presence of all PDE isoenzymes, except PDE2, via selective expression of 18 of 24 PDE genes in human ASM cells derived from donors with asthma and fatal asthma. ${ }^{17}$ Experimental studies indicate a possible role in asthma mainly for PDE3, PDE4, PDE5 and PDE7. Also, PDE8 expression and function in ASM have been documented. ${ }^{17}$

PDE3 is the predominant PDE isoenzyme in ASM and its inhibition produces ASM relaxation as well as enhancement of relaxation evoked by $\beta_{2}$-AR stimulation. ${ }^{7,28}$ However, PDE3 deficiency was shown to reduce allergic airway inflammation and to improve airway mucosal barrier function in allergic airway models. ${ }^{29}$ There is evidence that this isoenzyme plays also a critical role in basophil and mast cell degranulation. ${ }^{30}$

PDE4 isoenzyme is the principal isoenzyme in the majority of inflammatory cells of importance in the pathogenesis of asthma. It is highly expressed in inflammatory cells including T cells, macrophages, eosinophils and neutrophils and can degrade cAMP. ${ }^{28}$ It is also present in ASM cells, but its inhibition has not demonstrated acute bronchodilator effects in humans. ${ }^{31}$ PDE4 activity is also predominant in ciliary epithelia. ${ }^{23}$

PDE5 has a relatively high expression level in vascular smooth muscle cells but it is also widely expressed in ASM, bronchial epithelial cells and lung fibroblasts. ${ }^{18}$ PDE5 inhibition and the subsequent cGMP increase without any significant effect on cAMP caused protein kinase G (PKG)dependent smooth muscle relaxation ${ }^{32}$ and a relaxation of guinea pigs' trachea in vitro and a bronchodilatory activity in vivo. ${ }^{33}$ Increased intracellular levels of cGMP also led to suppressed immunological responses. ${ }^{34}$ The significant suppression of both in vivo and in vitro contractile responses to the cumulative doses of acetylcholine and histamine in ovalbumin-sensitized animals was associated with decreased numbers of circulating leukocytes and eosinophils and concentrations of interleukin (IL)-4, IL-5 and TNF- $\alpha$ in the lung homogenate. ${ }^{35}$ However, Banner and Page failed to show any significant effects of acute or chronic administration of PDE5 inhibitor in ovalbumininduced eosinophil infiltration in guinea pigs. ${ }^{36}$

PDE7 is present in the soluble fraction of $\mathrm{T}$ cells, both $\mathrm{CD}^{+}$and $\mathrm{CD}^{+}$, and controls intracellular levels of cAMP. $^{37}$ It has been suggested that PDE7 may play a crucial role in $\mathrm{T}$ cell activity. ${ }^{38}$ Nevertheless, a lack of involvement of PDE7 in airway hyperreactivity and airway inflammation was observed in an experimental model of asthma with sensitised mice. ${ }^{39}$

PDE8 is a less widely expressed form of PDE, but has 40-100-fold greater affinity for cAMP than PDE4. ${ }^{40}$ It is localized in lipid rafts and specifically regulates cAMP signaling stimulated by $\beta_{2}$-AR with no effect on cAMP signaling stimulated by prostaglandin $\mathrm{E}_{2}$ in $\mathrm{ASM}^{18}$ It has been suggested that PDE8 might also serve as a novel and promising target for inhibition of activated T-lymphocyte migration from the bloodstream into the tissue during the inflammatory response. ${ }^{41}$

These findings have led to the investigation of the PDE family as a potential target in the treatment of asthma and to explore the possibility of developing drugs with the ability not only to inhibit single PDEs but also to interact simultaneously with different PDEs. ${ }^{20}$

\section{Current Scenario with Selective PDE Inhibitors in Asthma}

Experimental and clinical trials with PDE inhibitors in asthma treatment have already been discussed in some recent review articles. ${ }^{8-10}$ These articles reported that the potential therapeutic utility of PDE inhibitors has been demonstrated in various animal models of asthma and also in clinical trials. In particular, the ability of PDE4 inhibitors to inhibit important characteristics of asthma, ie, eosinophil recruitment to the airways and bronchial hyperresponsiveness has been repeatedly documented. ${ }^{8-10}$ Roflumilast was found unable to exert any effect on the acute phase response (bronchoconstriction), but it reduced the late phase asthmatic response and prevented the subsequent increase in bronchial reactivity following an allergen challenge. ${ }^{33}$ Furthermore, it consistently improved lung function of asthmatic patients when combined with ICS or montelukast. ${ }^{42}$ A series of PDE4 inhibitors, such as cilomilast, revamilast, MEM 1414 and BLX-028914, reached the clinical stage in asthma, but their development was abandoned. ${ }^{8}$ In effect, their clinical 
efficacy was restricted by the dose-limiting side effects. Adverse gastrointestinal effects including nausea, emesis, and diarrhoea are the major side effects of PDE4 inhibitors that limit their tolerability. ${ }^{8-10}$

Consequently, no PDE4 inhibitor has been approved for the treatment of asthma, although a systematic review and meta-analysis of major databases (1946-2016) concluded that in patients with mild asthma, oral PDE4 inhibitors can be considered as an alternative treatment to regular bronchodilators and inhaled controllers. ${ }^{43}$ In any case, a clinical trial is evaluating the effects of roflumilast on restoring response to $\beta_{2}$-agonists in Type- 2 low (non-T2) obese asthmatics (ClinicalTrials.gov Identifier: NCT04108377).

Also PDE3 inhibitors have been shown to counteract early and late airway responses induced by allergens ${ }^{44}$ and to cause bronchodilation in patients with asthma. ${ }^{8}$ However, there has been no real development for these drugs as it is suspected that they might induce adverse events, particularly in the cardiovascular system because PDE3 participates in the regulation of vascular smooth muscle and cardiac muscle function. ${ }^{45}$ Nevertheless, intravenous enoximone, which is a selective PDE3 inhibitor used for treatment of heart failure, worked instantaneously resolving bronchospasm, regardless of the severity of the asthma or the dosage, in eight patients. ${ }^{46}$ Also oral enoximone very quickly and significantly improved difficult-to-treat asthma, as well as reduced steroid and $\beta_{2}$-agonist drugs in 47 out of 51 adult patients, and in 11 of the 12 children. $^{47}$

Preclinical data have shown that sildenafil, a selective PDE5 inhibitor that is used extensively in erectile dysfunction in males, inhibits the airway hypersensitivity and leukocyte influx in conscious guinea pigs ${ }^{48}$ and could reduce airway inflammation and mucus production in rats. ${ }^{49}$ Furthermore, sildenafil and tadalafil, another selective PDE5 inhibitor decreased pro-inflammatory cytokines in serum and BALF and inhibited oxidative and nitrosative stress in a rat model of bronchial asthma. ${ }^{50}$ However, a pilot study conducted for investigating the effect of sildenafil on pulmonary function in patients with severe asthma concluded that this PDE5 inhibitor does not improve the severity of asthma and the quality of life. ${ }^{51}$

\section{Looking for a Future Scenario with Selective PDE4 Inhibitors in Asthma} The PDE4 family comprises four genes, PDE4A, PDE4B, PDE4C, and PDE4D. ${ }^{52}$ Apparently, inhibition of PDE4D is responsible for the emetic effects of PDE4 inhibitors. This has led to significant efforts to develop PDE4 inhibitors selective for PDE4B over PDE4D in expectation of an improved safety profile and also considering the predominance of the PDE4B subtype in inflammatory cell regulation. ${ }^{53}$ In effect, there are data suggesting that PDE4B is a vital target in T2-cell function and in the development of airway hyperresponsiveness in allergic asthma. ${ }^{21}$ Unfortunately, no selective oral PDE4B inhibitors have advanced to clinical trials. ${ }^{54}$ The conformation of Met431 of PDE4B is the only minor difference between the active sites of PDE4B and PDE4D. ${ }^{55}$ This makes it difficult to design PDE4 subfamily selective inhibitors against only the catalytic domain of PDE4. In any case, there are studies that indicate that inhibition of PDE4D may not be a critical factor to elicit emesis. ${ }^{56}$

Inhaled administration represents another potential approach to improve the therapeutic index of PDE4 inhibitors because it reduces systemic exposure and, likely, associated side effects. ${ }^{8}$

Clinical trials in asthma with AWD-12-281 and tofimilast have been failed to demonstrate efficacy and development have been discontinued. ${ }^{57}$ Conversely, GSK256066, a selective PDE4B inhibitor that can be delivered by inhalation, demonstrated a protective effect on both early and late responses to inhaled allergen. ${ }^{58}$ However, it had no effect on the secondary endpoints of methacholine reactivity post-allergen challenge or exhaled nitric oxide. In any case, no further clinical information on the effects of GSK256066 in asthma is available to date. It seems probable that its clinical development has been stopped because of the low solubility and the lipophilic nature of GSK256066 causing too low concentrations of free compound in the lung to exert a pharmacological effect. ${ }^{59}$

Clinical development of tanimilast (CHF6001), which has been designed for treating pulmonary inflammatory diseases via inhaled administration, has so far been more consistent although mainly focused on its use in COPD. ${ }^{60}$ It inhibits PDE4 isoforms A-D with equal potency and is 7-fold more potent than roflumilast in inhibiting PDE4 enzymatic activity. ${ }^{61}$ Tanimilast reduced the allergen challenge response in asthmatic patients $^{62}$ and inhibited IFN $\gamma$, IL-2 and IL-17, but not IL-13, secretion from both mild and moderate asthma patient BAL cells, showing a greater effect than corticosteroids on the $\mathrm{T} 1$ response. $^{63}$ 


\section{Novel Prospective with PDE Inhibitors in Asthma}

Targeting PDE4 alone may not fully resolve airway inflammation. As already mentioned, different PDE isozymes selectively regulate cAMP or cGMP signaling in different subcellular microdomains. It is likely that individual PDEs are involved in specific locations at certain time-points based on different stimulations/activations, ${ }^{17,18}$ and furthermore, multiple PDE variants participate in the complex signaling network involving central regulatory mechanisms. ${ }^{18,64}$ Therefore, targeting different PDE enzymes with dual PDE inhibitors may be required for optimal anti-inflammatory action, ${ }^{20,65}$ which is fundamental in asthma. The possibility that inhibition of PDE4 enzymes together with a second family of PDEs could provide a therapeutic benefit at a concentration that does not cause vomiting is also not negligible. ${ }^{66}$ Another potential option is to develop molecules specifically designed to have multivalent (multifunctional) ligands, which contain two or more pharmacophores, ${ }^{20,65}$ socalled hybrid molecules.

The practical application of these concepts has led to the development of drugs capable of interacting simultaneously with different PDEs.

\section{Dual PDE3/PDE4 Inhibitors}

PDE3 and PDE4 are important in influencing cAMP signaling, ${ }^{67}$ but it is likely that they can regulate different pools of cAMP since PDE3 is predominantly localized in the particle cell fraction and PDE4 is predominantly cytosolic. ${ }^{68}$ Dual inhibitory activity for both PDE3 and PDE4 in the same molecule increases intracellular cAMP levels and allows obtain both bronchodilator and antiinflammatory activity, ${ }^{20,65}$ and improves endothelial and epithelial barrier function. ${ }^{69}$ It has been suggested that in addition to targeting PDE4 to reduce allergic airway inflammation, targeting PDE3 also considerably decreases allergic airway inflammation. ${ }^{70}$ Furthermore, in asthma dual PDE3/PDE4 inhibitors may enhance, sensitize, and prolong the effects of LABA/ICS combination therapies ${ }^{71}$ Nonetheless, zardaverine, benzafentrine, tolafentrine and pumafentrine that selectively inhibit PDE3 and PDE4 have not progressed to the clinic presumably because of lack of efficacy or concerns about safety. ${ }^{18}$

Ensifentrine (RPL554) is reported as an inhaled PDE3/4 inhibitor, although its affinity for PDE3 is 3,440 times higher than that for PDE4. ${ }^{72}$ It inhibited the contractile tone induced by electrical field stimulation in sensitized human bronchi, which represents a reliable ex-vivo model of asthma. ${ }^{73}$ Furthermore, a synergistic interaction in relaxing both human medium and small isolated bronchi, in terms of peak relaxation and an extended duration of action was documented when ensifentrine was administered in combination with a long-acting muscarinic antagonist. ${ }^{74}$ At present, it is the only drug designed to function as a dual PDE3/4 inhibitor that is in clinical trials for airway diseases. A treatment with an old nebulized formulation of ensifentrine at a dose of $0.018 \mathrm{mg} / \mathrm{kg}$ given for 6 consecutive days in subjects with clinically stable asthma induced a large and persistent improvement in forced expiratory volume in one second that was better than broncholytic effect induced salbutamol $200 \mu \mathrm{g} .^{75}$ A new inhaled suspension formulation administered as single doses $(0.4 \mathrm{mg}$, $1.5 \mathrm{mg}, 6 \mathrm{mg}, 24 \mathrm{mg}$ ) in patients with mild to moderate chronic asthma showed a pharmacokinetic profile that was similar to that observed in healthy subjects. ${ }^{70}$ Additionally, it induced a dose-dependent bronchodilation and was as effective as a therapeutic dose of nebulised salbutamol. ${ }^{76}$

Recently, it has been shown that synthetic hesperetin5,7,3'-O-trimethylether dually inhibited PDE3/4 and induced anti-inflammatory and bronchodilator actions in a murine model of asthma, including suppression of airway hyperresponsiveness, and attenuation of inflammatory cells and cytokines, with the potential of causing few or no gastrointestinal adverse effects. ${ }^{77}$

\section{Dual PDE4/PDE5 Inhibitors}

Simultaneous PDE4 and PDE5 blockade could act at multiple levels in airway disorders. In fact, it may not only decrease pulmonary arterial hypertension but also reduce lung inflammation and possibly remodeling and, at the same time, improve lung function. ${ }^{66}$ A combination of tadalafil, a selective inhibitor of PDE5, and roflumilast caused the suppression of airway reactivity and markers of inflammation in the model of ovalbumin-induced eosinophilic inflammation associated with the airway hyperresponsiveness, which was comparable to the effects of dexamethasone at the same dose. ${ }^{78}$ However, no additive effect compared to PDE4 inhibition alone was observed when the combination was administered at a reduced dose. Despite these interesting preclinical findings, there is currently no bifunctional PDE4/PDE5 inhibitor in clinical development, and this not only because selective PDE5 inhibitors are not effective in asthma, but also because of the potential risk of a worsening of gas exchange that they can induce. $^{79}$ 


\section{Dual PDE4/PDE7 Inhibitors}

The inhibition of PDE7 is unable to influence proinflammatory cells per se but increases the inhibitory effect of other cAMP-elevating drugs. ${ }^{80}$ The co-expression of PDE4 and PDE7 in most immunoinflammatory cells has generated speculation that dual inhibition of PDE7 and PDE4 could be an effective strategy to treat asthma. ${ }^{6}$ In fact, simultaneous inhibition of PDE4 and PDE7 boosts the effects detected after the inhibition of PDE4 or PDE7 alone ${ }^{81}$ and suppresses airway hyperresponsiveness. ${ }^{82}$ A relaxing effect of combined PDE4/PDE7 inhibition (rolipram plus BRL50481) on acetylcholine-induced lung and airway contraction was documented in ovalbumin-sensitized guinea pigs. ${ }^{83}$ A study aimed to characterize the pharmacological profile of YM-393059, which is a dual PDE4/PDE7 inhibitor, showed that this bifunctional drug is able to inhibit both T1- and T2-cell-dependent reactions and also the function of neutrophils, and tends to reduce the serum immunoglobulin E antibody level. ${ }^{83} \mathrm{~T}-2585$, and IR284 are other dual PDE4/PDE7 inhibitors that revealed anti-inflammatory activity, ${ }^{84}$ although the inhibition of PDE4 could influence ASM tone. Recently, several new potential PDE4/PDE7 inhibitors, such as a series of butanehydrazide derivatives of purine-2,6-dione, ${ }^{85}$ a series of novel amide derivatives of 1,3-dimethyl-2,6-dioxopurin-7-ylalkylcarboxylic acids, ${ }^{86}$ and some newer substituted 1,3-thiazolidine-2,4-dione derivatives $^{87}$ have been designed and synthesised. However, the real value of these compounds in the treatment of asthma is still unknown, and moreover, we do not know which of them will really be evaluated in humans.

\section{Dual PDE4/PDEI Inhibitors}

The PDE1 family is regulated by allosteric interactions with the calcium calmodulin complex $\left(\mathrm{Ca}^{2+}-\mathrm{CaM}\right) .{ }^{88}$ The binding of one $\mathrm{Ca}^{2+}-\mathrm{CaM}$ complex per monomer to binding sites near the $\mathrm{N}$ terminus stimulates cyclic nucleotide hydrolysis. There are three PDE1 isoforms, PDE1A, PDE1B, and PDE1C. PDE1C, which degrades both cAMP and cGMP with high affinity, is widely expressed in airway myocytes ${ }^{89}$ and is a major regulator of smooth muscle proliferation. ${ }^{88}$ Airway remodelling (thickening, hyperplasia, and hypertrophy) is characteristic of asthma. ${ }^{90}$ It is therefore logical that dual-specificity inhibitors of PDE1C and PDE4 might selectively target proliferating ASM cells and influence the remodelling process and inflammation and have utility in the treatment of patients with asthma. KF19514 is a dual PDE4/PDE1 inhibitor that exhibited a potent anti-inflammatory effect in animal models of acute allergen challenge for inhibiting antigen-induced bronchospasm. ${ }^{91}$ It was also shown that KF19514 suppressed inflammation and arrest airway remodelling in a murine model of chronic asthma. ${ }^{92}$ Thus, simultaneous inhibition of PDE1c and PDE4 could be seen as a potential drug target in asthma treatment, but more precise studies are necessary in both preclinical and, mainly, clinical research.

\section{Dual PDE Inhibitors That Also Inhibit PDE8}

The utility of PDE8 inhibitors alone or in combination with PDE4 or PDE7 inhibitors has also been claimed to treat inflammation and immune-related disorders. ${ }^{93}$

So far, are only two dual PDE inhibitors, dipyridamole, which is a PDE5/PDE8 inhibitor that inhibits platelet aggregation, and $\mathrm{BC} 8-15$, which is a PDE4/PDE8 inhibitor. However, dipyridamole also increases cAMP levels by blocking uptake of adenosine and enhances adenosineinduced bronchoconstriction in asthmatic subjects without changes in baseline airway caliber. ${ }^{94}$ The PDE8 selective inhibitor PF-4957325 has been widely used in PDE8 research but not in asthma.

\section{Pan-PDE Inhibitors}

There is currently interest in developing pan-PDE inhibitors, which are capable of inhibiting various isoforms within different PDE classes. This approach would allow not only inducing synergistic effects deriving from targeting multiple PDE subtypes simultaneously, ${ }^{95}$ but also to eventually overcome the changes in the expression of the single PDEs that may arise in the course of asthma. ${ }^{18}$ A group of potent, pan-PDE inhibitors from the group of 7.8-disubstituted purine-2,6-dione derivatives represent promising anti-remodelling drug candidates for further research. ${ }^{96}$

\section{Dual PDE4 Inhibitors $/ \beta_{2}$-Agonists}

It has been suggested that fusing the head group of an existing long-acting $\beta_{2}$-agonist (LABA) to a variety of structurally dissimilar PDE4 inhibitors could provide additive or synergistic bronchodilation and anti-inflammatory activity in the lung. ${ }^{97}$ The potential advantage of these compounds is that both $\beta_{2}$-agonists and PDE4 inhibitors rely on modulation of cAMP to elicit their effects. ${ }^{98}$ 
GS-5759 is a dual PDE4 inhibitor $/ \beta_{2}$-agonist made by linking a GSK256066 structurally related PDE4 inhibitor to an indacaterol structurally related $\beta_{2}$-agonist. ${ }^{99}$ It is a potent relaxer of airway smooth muscle strips and has both anti-inflammatory and antifibrotic activity in a variety of cell types ${ }^{100}$ and demonstrated a dose-related bronchodilation in guinea pigs, dogs, and cynomolgus monkeys. ${ }^{101}$ However, it has no clinical development reported.

Also, a series of molecules that combine the pharmacophores of the PDE4 inhibitor phthalazinone or roflumilast and salmeterol ${ }^{102}$ or pharmacophores of phthalazinone and formoterol ${ }^{103}$ have been described.

\section{Dual PDE4 Inhibitors/Muscarinic Antagonists}

Exploring the structure-activity relationships around the 4.6-diaminopyrimidine scaffold led to the identification of the dual PDE4 inhibitor/muscarinic antagonist UCB $-101333-3,{ }^{104}$ which decreased inflammatory cells and murine keratinocyte level (equivalent to IL-8 in humans) in BAL fluid of mice when given by nose-only aerosol. ${ }^{105}$ It was assumed that the addition of an ultra-LABA to UCB-101333-3 should create a potent combination for treating asthma, ${ }^{106}$ but apparently the development of this bifunctional drug has been stopped.

Afterwards, dual PDE4 inhibitors/muscarinic antagonists that utilize a pyrazolopyridine as the PDE4 inhibitor and a biaryl-containing muscarinic antagonist but differ in the linker used to combine these two activities into the same molecule were identified. ${ }^{107}$ However, even for these molecules, it seems that the clinical development has not begun so far.

\section{Conclusion}

Notwithstanding the presence in the literature of many data indicating the potential benefits of PDE inhibitors as an add-on treatment especially in severe asthma due to their bronchodilator and/or anti-inflammatory activity, no compound has yet reached the market as asthma treatment. However, in recent years, there has been a growing interest in developing new PDE inhibitors with an improved safety profile, possibly also due to the size of the target patient population that would benefit from another antiinflammatory drug and the high costs for the production of new biological drugs. ${ }^{10}$ In particular, the research focused on the development of drugs capable of interacting simultaneously with different PDEs but, although there are several new agents with interesting pharmacological profiles, CHF 6001 and RPL554 are still the only molecules under clinical development.

\section{Expert Opinion}

It is increasingly accepted that the term asthma is an umbrella term that is used to give a simplified definition of grouped clinical and physiological characteristics (phenotypes) and distinct mechanistic pathways (endotypes). ${ }^{108}$ However, although asthma is a highly heterogeneous condition, this has not been accompanied by the concrete identification of heterogeneity-focused treatments. ${ }^{109}$

As already mentioned, the superfamily of PDEs can regulate a wide range of biological functions. ${ }^{18}$ It obvious, therefore, that PDEs are attractive pharmaceutical targets for a personalized asthma treatment.

It is likely that in the future, the application of omics data will allow identifying the specific PDEs involved in the pathological process in each patient. However, this is a rather complicated and expensive approach. At present time, a precision medicine strategy for the management of patients with airway disease that is free from the traditional diagnostic labels and based on identifying "treatable traits" in each patient is still preferable. ${ }^{110}$ Fixed airflow limitation, bronchodilator reversibility, Type-2 inflammation, neutrophilic inflammation, cough, exercise-induced respiratory symptoms, and bronchitis are the most prevalent traits in severe asthma. ${ }^{111}$ The data currently available suggest that the use of PDE inhibitors, mainly if administered as dual PDE inhibitor, is capable of influencing practically all these traits. However, there is an absolute need to confirm the impact of such agents on individual treatable traits in specially designed studies.

In our article published in 2014, we highlighted the need to test roflumilast in the group of asthmatics, especially those suffering from the severe form of asthma, in which the neutrophilic inflammation predominates and inhaled corticosteroid are not as effective. ${ }^{8}$ Experimental data indicate that administration of roflumilast attenuates airway inflammation and airway hyperresponsiveness in neutrophilic asthma model and adding roflumilast to dexamethasone results in further beneficial effect. ${ }^{112}$ Using data from eight placebo-controlled, double-blind Phase IIII studies, significant reductions in allergen-induced airway inflammation, including a reduction in neutrophil count, were observed. ${ }^{113}$ Unfortunately, no specific study has been conducted so far to verify whether PDE4 inhibitors provide benefit in severe asthma patients characterized 
by neutrophilic inflammation. Additionally, no trials exist to date evaluating the use of roflumilast or other PDE inhibitors in patients with overlapping characteristics of asthma and COPD, but PDE4 inhibitors could be considered, particularly among those individuals with frequent exacerbations.

There is also the urgent need to understand if the use of PDE inhibitors impacts on Type-2 high or T2 asthma, which is characterized by increased eosinophilia and other signs of type- 2 airway inflammation, including increased numbers of airway mast cells subgroups, in a different way compared to Type-2 low or non-T2 asthma, which is characterized by levels of type 2 inflammation in the airways comparable to those found in healthy subjects. ${ }^{114}$ However, it is likely that specific dual PDE inhibitors may be more effective than others against a specific type-2 asthma. In any case, both dual PDE inhibitors and hybrid molecules with other bronchodilators may serve as a basis for improved "multiple-therapy" fixed-dose combination inhalers through co-formulation that could deliver three or even four complementary therapeutic effects for patients with asthma.

\section{Authorship}

All authors made substantial contributions to conception and design, acquisition of data, or analysis and interpretation of data; took part in drafting the article or revising it critically for important intellectual content; agreed to submit to the current journal; gave final approval of the version to be published; and agree to be accountable for all aspects of the work.

\section{Funding}

There is no funding to report.

\section{Disclosure}

MGM reports personal fees from Almirall and AstraZeneca, grants from GlaxoSmithKline and Novartis; has participated as a speaker and advisor in scientific meetings and courses under the sponsorship of Almirall, AstraZeneca, Boehringer Ingelheim, Chiesi Farmaceutici, GlaxoSmithKline, Novartis, and Zambon; she is or has been a consultant to ABC Farmaceutici, and Chiesi Farmaceutici; and her department was funded by GlaxoSmithKline, and Novartis. JO reports personal fees from Astrazeneca and has participated as a speaker in scientific meetings and courses under the sponsorship of AstraZeneca, Boehringer Ingelheim, Chiesi Farmaceutici, GlaxoSmithKline, Menarini Group, Novartis, and
Zambon. FC declares no conflicts of interest. PR reports grants and personal fees from Almirall, AstraZeneca, Biofutura, Boehringer Ingelheim, Chiesi Farmaceutici, GlaxoSmithKline, Menarini Group, Mundipharma, and Novartis; has participated as a speaker, and advisor in scientific meetings and courses under the sponsorship of Almirall, AstraZeneca, Biofutura, Boehringer Ingelheim, Chiesi Farmaceutici, GlaxoSmithKline, Menarini Group, Mundipharma, and Novartis; and her department was funded by Almirall, Boehringer Ingelheim, Novartis, and Zambon (during the study and outside the submitted work). MC reports personal fees from Edmond Pharma, grants from Almirall, Menarini Group and Novartis; her department was funded by Almirall, Boehringer Ingelheim, Novartis, and Zambon; has participated as a faculty member and advisor in scientific meetings and courses under the sponsorship of Almirall, AstraZeneca, Biofutura, Boehringer Ingelheim, Chiesi Farmaceutici, Cipla, Edmond Pharma, GlaxoSmithKline, Lallemand, Menarini Group, Mundipharma, Novartis, Pfizer, Teva, Verona Pharma, and Zambon he is or has been a consultant to ABC Farmaceutici, AstraZeneca, Chiesi Farmaceutici, Edmond Pharma, Lallemand, Novartis, Ockham Biotech, Verona Pharma, and Zambon. The authors report no other potential conflicts of interest for this work.

\section{References}

1. Matera MG, Page C, Cazzola M. Doxofylline is not just another theophylline! Int J Chron Obstruct Pulmon Dis. 2017;12:3487-3493. doi:10.2147/COPD.S150887

2. Barnes PJ. Theophylline. Am J Respir Crit Care Med. 2013;188 (8):901-906. doi:10.1164/rccm.201302-0388PP

3. Boswell-Smith V, Cazzola M, Page CP. Are phosphodiesterase 4 inhibitors just more theophylline? J Allergy Clin Immunol. 2006;117 (6):1237-1243. doi:10.1016/j.jaci.2006.02.045

4. Torphy TJ, Undem BJ. Phosphodiesterase inhibitors: new opportunities for the treatment of asthma. Thorax. 1991;46(7):512-523. doi:10.1136/thx.46.7.512

5. Cazzola M, Calzetta L, Rogliani P, Matera MG. The discovery of roflumilast for the treatment of chronic obstructive pulmonary disease. Expert Opin Drug Discov. 2016;11(7):733-744. doi:10. 1080/17460441.2016.1184642

6. Chung FK. Phosphodiesterase inhibitors in airways disease. Eur $J$ Pharmacol. 2006;533(1-3):110-117. doi:10.1016/j.ejphar.2005. 12.059

7. Page CP. Phosphodiesterase inhibitors for the treatment of asthma and chronic obstructive pulmonary disease. Int Arch Allergy Immunol. 2014;165(3):152-164. doi:10.1159/000368800

8. Matera MG, Page C, Cazzola M. PDE inhibitors currently in early clinical trials for the treatment of asthma. Expert Opin Investig Drugs. 2014;23 (9):1267-1275. doi:10.1517/13543784.2014.921157

9. Ntontsi P, Detta A, Bakakos P, Loukides S, Hillas G. Experimental and investigational phosphodiesterase inhibitors in development for asthma. Expert Opin Investig Drugs. 2019;28(3):261-266. doi:10.1080/13543784.2019.1571582 
10. Al-Sajee D, Yin X, Gauvreau GM. An evaluation of roflumilast and PDE4 inhibitors with a focus on the treatment of asthma. Expert Opin Pharmacother. 2019;20(5):609-620. doi:10.1080/ 14656566.2019.1570132

11. Shastri MD, Chong WC, Dua K, et al. Emerging concepts and directed therapeutics for the management of asthma: regulating the regulators. Inflammopharmacology. 2020. doi:10.1007/s107 87-020-00770-y

12. Meltzer EO, Chervinsky P, Busse W, et al. Roflumilast for asthma: efficacy findings in placebo-controlled studies. Pulm Pharmacol Ther. 2015;35(Suppl):S20-S27. doi:10.1016/j.pupt.20 15.10.006

13. Reddy AT, Lakshmi SP, Banno A, Reddy RC. Glucocorticoid receptor $\alpha$ mediates roflumilast's ability to restore dexamethasone sensitivity in COPD. Int $J$ Chron Obstruct Pulmon Dis. 2020;15:125-134. doi:10.2147/COPD.S230188

14. Maselli DJ, Hanania NA. Management of asthma COPD overlap. Ann Allergy Asthma Immunol. 2019;123(4):335-344. doi:10.10 16/j.anai.2019.07.021

15. Albertson TE, Chenoweth JA, Pearson SJ, Murin S. The pharmacological management of asthma-chronic obstructive pulmonary disease overlap syndrome (ACOS). Expert Opin Pharmacother. 2020;21(2):213-231. doi:10.1080/14656566.2019.1701656

16. Maurice DH, Ke H, Ahmad F, Wang Y, Chung J, Manganiello VC. Advances in targeting cyclic nucleotide phosphodiesterases. Nat Rev Drug Discov. 2014;13(4):290-314.

17. Johnstone TB, Smith KH, Koziol-White CJ, et al. PDE8 is expressed in human airway smooth muscle and selectively regulates cAMP signaling by $\beta_{2}$-adrenergic receptors and adenylyl cyclase 6. Am J Respir Cell Mol Biol. 2018;58(4):530-541. doi:10.1165/rcmb.2017-0294OC

18. Zuo H, Cattani-Cavalieri I, Musheshe N, Nikolaev VO, Schmidt M. Phosphodiesterases as therapeutic targets for respiratory diseases. Pharmacol Ther. 2019;197:225-242.

19. MarshallRaj G, Priyadarshini R. Phosphodiesterase: what is in an enzyme? J Biomed Pharmacol Res. 2015;4(5):20-31.

20. Page C, Cazzola M. Bifunctional drugs for the treatment of asthma and chronic obstructive pulmonary disease. Eur Respir J. 2014;44(2):475-482. doi:10.1183/09031936.00003814

21. Jin SL, Goya S, Nakae S, et al. Phosphodiesterase 4B is essential for $\mathrm{T}_{\mathrm{H}}$ 2-cell function and development of airway hyperresponsiveness in allergic asthma. J Allergy Clin Immunol. 2010;126 (6):1252-1259. doi:10.1016/j.jaci.2010.08.014

22. Page CP, Selective SD. PDE inhibitors as novel treatments for respiratory diseases. Curr Opin Pharmacol. 2012;12(3):275-286. doi:10.1016/j.coph.2012.02.016

23. Joskova M, Mokry J, Franova S. Respiratory cilia as a therapeutic target of phosphodiesterase inhibitors. Front Pharmacol. 2020; 11:609. doi:10.3389/fphar.2020.00609

24. Thomas B, Rutman A, Hirst RA, et al. Ciliary dysfunction and ultrastructural abnormalities are features of severe asthma. J Allergy Clin Immunol. 2010;126(4):722-729. doi:10.1016/j.jaci 2010.05.046

25. Stasch JP, Pacher P, Evgenov OV. Soluble guanylate cyclase as an emerging therapeutic target in cardiopulmonary disease. Circulation. 2011;123(20):2263-2273. doi:10.1161/CIRCULATIONAHA.110. 981738

26. Lam M, Bourke JE. A new pathway to airway relaxation: targeting the "other" cyclase in asthma. Am J Respir Cell Mol Biol. 2020;62(1):3-4. doi:10.1165/rcmb.2019-0274ED

27. Ghosh A, Koziol-White CJ, Jester WF, et al. An inherent dysfunction in soluble guanylyl cyclase is present in the airway of severe asthmatics and is associated with aberrant redox enzyme expression and compromised NO-cGMP signaling. Redox Biol. 2021;39:101832. doi:10.1016/j.redox.2020.101832
28. Mokry J, Mokra D. Immunological aspects of phosphodiesterase inhibition in the respiratory system. Respir Physiol Neurobiol. 2013;187(1):11-17. doi:10.1016/j.resp.2013.02.004

29. KleinJan A. Airway inflammation in asthma: key players beyond the Th2 pathway. Curr Opin Pulm Med. 2016;22(1):46-52. doi:10.1097/MCP.0000000000000224

30. Beute J, Ganesh K, Nastiti H, et al. PDE3 inhibition reduces epithelial mast cell numbers in allergic airway inflammation and attenuates degranulation of basophils and mast cells. Front Pharmacol. 2020;11:470. doi:10.3389/fphar.2020.00470

31. Boswell-Smith V, Spina D, Oxford AW, Comer MB, Seeds EA, Page CP. The pharmacology of two novel long-acting phosphodiesterase 3/4 inhibitors, RPL554 [9,10-dimethoxy-2(2,4,6-trimethylphenylimino)-3-(n-carbamoyl-2-aminoethyl)-3,4,6,7-tetrahydro-2H-pyrimido[6,1-a]isoquinolin-4-one] and RPL565 [6,7-dihydro-2-(2,6-diisopropylphenoxy)-9,10-dimethoxy-4Hpyrimido[6,1-a]isoquinolin-4-one]. $J$ Pharmacol Exp Ther. 2006;318(2):840-848. doi:10.1124/jpet.105.099192

32. Nijkamp FP, Folkerts G. Nitric oxide and bronchial hyperresponsiveness. Arch Int Pharmacodyn Ther. 1995;329 (1):81-96.

33. Kapui Z, Schaeffer P, Mikus EG, et al. Experimental studies on guanosine 3',5'-cyclic monophosphate levels and airway responsiveness of the novel phosphodiesterase type 5 inhibitor SR 265579 in guinea-pigs. Arzneimittelforschung. 1999;49(8):68 5-693.

34. Boswell-Smith V, Spina D, Page CP. Phosphodiesterase inhibitors. Br J Pharmacol. 2006;147(Suppl 1):S252-257. doi:10. 1038/sj.bjp.0706495

35. Urbanova A, Medvedova I, Kertys M, et al. Dose dependent effects of tadalafil and roflumilast on ovalbumin-induced airway hyperresponsiveness in guinea pigs. Exp Lung Res. 2017;43(9-10):407-416. doi:10.1080/01902148.2017.1386735

36. Banner KH, Page CP. Acute versus chronic administration of phosphodiesterase inhibitors on allergen-induced pulmonary cell influx in sensitized guinea-pigs. $\mathrm{Br} J$ Pharmacol. 1995;114 (1):93-98. doi:10.1111/j.1476-5381.1995.tb14910.x

37. Giembycz MA, Corrigan CJ, Seybold J, Newton R, Barnes PJ. Identification of cyclic AMP phosphodiesterases 3, 4 and 7 in human CD4+ and CD8+ T-lymphocytes: role in regulating proliferation and the biosynthesis of interleukin-2. $\mathrm{Br} J$ Pharmacol. 1996;118(8):1945-1958. doi:10.1111/j.1476-5381.1996.tb15629.x

38. Nakata A, Ogawa K, Sasaki T, et al. Potential role of phosphodiesterase 7 in human $\mathrm{T}$ cell function: comparative effects of two phosphodiesterase inhibitors. Clin Exp Immunol. 2002;128 (3):460-466. doi:10.1046/j.1365-2249.2002.01856.x

39. Chevalier E, Lagente V, Dupont M, et al. Lack of involvement of type 7 phosphodiesterase in an experimental model of asthma. Eur Respir J. 2012;39(3):582-588. doi:10.1183/09031936.00 102610

40. Vang AG, Basole C, Dong H, et al. Differential expression and function of PDE8 and PDE4 in effector T cells: implications for PDE8 as a drug target in inflammation. Front Pharmacol. 2016;7:259. doi:10.3389/fphar.2016.00259

41. Vang AG, Ben-Sasson SZ, Dong H, et al. PDE8 regulates rapid Teff cell adhesion and proliferation independent of ICER. PLoS One. 2010;5(8):e12011. doi:10.1371/journal.pone.0012011

42. Bodkhe S, Nikam M, Sherje AP, Khan T, Suvarna V, Patel K. Current insights on clinical efficacy of roflumilast for treatment of COPD, asthma and ACOS. Int Immunopharmacol. 2020;88: 106906. doi:10.1016/j.intimp.2020.106906

43. Luo J, Yang L, Yang J, et al. Efficacy and safety of phosphodiesterase 4 inhibitors in patients with asthma: a systematic review and meta-analysis. Respirology. 2018;23(5):467-477. doi:10.11 11/resp. 13276 
44. Bardin PG, Dorward MA, Lampe FC, Franke B, Holgate ST. Effect of selective phosphodiesterase 3 inhibition on the early and late asthmatic responses to inhaled allergen. $\mathrm{Br} J$ Clin Pharmacol. 1998;45(4):387-391. doi:10.1046/j.1365-2125.1998. t01-1-00689.x

45. Matera MG, Page CP, Calzetta L, Rogliani P, Cazzola M. Pharmacology and therapeutics of bronchodilators revisited. Pharmacol Rev. 2020;72(1):218-252.

46. Beute J. Emergency treatment of status asthmaticus with enoximone. Br J Anaesth. 2014;112(6):1105-1108. doi:10.1093/ $\mathrm{bja} / \mathrm{aeu} 048$

47. Beute J. (Oral) enoximone in asthma. ERJ Open Res. 2020;6 (4):00319-2020. doi:10.1183/23120541.00319-2020

48. Toward TJ, Smith N, Broadley KJ. Effect of phosphodiesterase-5 inhibitor, sildenafil (Viagra), in animal models of airways disease. Am J Respir Crit Care Med. 2004;169(2):227-234. doi:10.1164/ rccm.200211-1372OC

49. Wang T, Liu Y, Chen L, et al. Effect of sildenafil on acrolein-induced airway inflammation and mucus production in rats. Eur Respir J. 2009;33(5):1122-1132. doi:10.1183/090319 36.00055908

50. Laxmi V, Gupta R, Bhattacharya SK, Ray A, Gulati K. Inhibitory effects of sildenafil and tadalafil on inflammation, oxidative stress and nitrosative stress in animal model of bronchial asthma. Pharmacol Rep. 2019;71(3):517-521. doi:10.1016/j.pharep.2019. 02.008

51. Borsi H, Raji H, Shoushtari MH, Tavakol H, Haghighizadeh MH, Mal-Amir MD. Investigating the effect of sildenafil on improving lung function and quality of life in the patients with severe asthma. J Family Med Prim Care. 2019;8(7):2361-2363. doi:10. 4103/jfmpc.jfmpc_334_19

52. Bolger GB, Conti M, Houslay MD. Cellular functions of PDE4 enzymes. In: Beavo JA, Francis SH, Houslay MD, editors. Cyclic Nucleotide Phosphodiesterases in Health and Disease. Boca Raton, FL: CRC Press; 2007:99-130.

53. Kranz M, Wall M, Evans B, et al. Identification of PDE4B over 4D subtype-selective inhibitors revealing an unprecedented binding mode. Bioorg Med Chem. 2009;17(14):5336-5341. doi:10.10 16/j.bmc.2009.03.061

54. Contreras S, Milara J, Morcillo E, Cortijo J. Selective Inhibition of Phosphodiesterases 4A, B, C and D isoforms in chronic respiratory diseases: current and future evidences. Curr Pharm Des. 2017;23(14):2073-2083. doi:10.2174/13816128236661702 14105651

55. Peng T, Qi B, He J, Ke H, Shi J. Advances in the development of phosphodiesterase-4 inhibitors. J Med Chem. 2020;63(19):10 594-10617. doi:10.1021/acs.jmedchem.9b02170

56. Burgin AB, Magnusson OT, Singh J, et al. Design of phosphodiesterase 4D (PDE4D) allosteric modulators for enhancing cognition with improved safety. Nat Biotechnol. 2010;28(1):63-70. doi:10.1038/nbt.1598

57. Singh D, Petavy F, Macdonald AJ, Lazaar AL, O'Connor BJ. The inhaled phosphodiesterase 4 inhibitor GSK256066 reduces allergen challenge responses in asthma. Respir Res. 2010;11(1):26. doi:10.1186/1465-9921-11-26

58. De Savi C, Cox RJ, Warner DJ, et al. Efficacious inhaled PDE4 inhibitors with low emetic potential and long duration of action for the treatment of COPD. J Med Chem. 2014;57(11):46 61-4676. doi:10.1021/jm5001216

59. Phillips JE. Inhaled phosphodiesterase 4 (PDE4) inhibitors for inflammatory respiratory diseases. Front Pharmacol. 2020;11: 259. doi:10.3389/fphar.2020.00259

60. Matera MG, Cazzola M, Page C. Prospects for COPD treatment. Curr Opin Pharmacol. 2020;56:74-84. doi:10.1016/j.coph.2020. 11.003
61. Moretto N, Caruso P, Bosco R, et al. CHF6001 I: a novel highly potent and selective phosphodiesterase 4 inhibitor with robust anti-inflammatory activity and suitable for topical pulmonary administration. J Pharmacol Exp Ther. 2015;352(3):559-567. doi:10.1124/jpet.114.220541

62. Singh D, Leaker B, Boyce M, et al. A novel inhaled phosphodiesterase 4 inhibitor (CHF6001) reduces the allergen challenge response in asthmatic patients. Pulm Pharmacol Ther. 2016; 40:1-6. doi:10.1016/j.pupt.2016.06.011

63. Southworth T, Kaur M, Hodgson L, et al. Anti-inflammatory effects of the phosphodiesterase type 4 inhibitor CHF6001 on bronchoalveolar lavage lymphocytes from asthma patients. Cytokine. 2019;113:68-73. doi:10.1016/j.cyto.2018.06.007

64. Cazzola M, Rogliani P, Matera MG. The future of bronchodilation: looking for new classes of bronchodilators. Eur Respir Rev. 2019;28(154):190095. doi:10.1183/16000617.0095-2019

65. Page C, Cazzola M. Bifunctional drugs for the treatment of respiratory diseases. Handb Exp Pharmacol. 2017;237:197-212.

66. Giembycz MA. Life after PDE4: overcoming adverse events with dual-specificity phosphodiesterase inhibitors. Curr Opin Pharmacol. 2005;5(3):238-244. doi:10.1016/j.coph.2005.04.001

67. Beute J, Manganiello V, Klein JA. Phosphodiesterase 3 and 4 inhibition: facing a bright future in asthma control. In: Huang HKG, Tsai CHS, editors. Asthma Diagnosis and Management - Approach Based on Phenotype and Endotype. IntechOpen; 2018. doi:10.5772/intechopen.74309

68. Matera MG, Page CP, Cazzola M. Novel bronchodilators for the treatment of chronic obstructive pulmonary disease. Trends Pharmacol Sci. 2011;32(8):495-506. doi:10.1016/j.tips.2011.04. 003

69. Abbott-Banner KH, Page CP. Dual PDE3/4 and PDE4 inhibitors: novel treatments for COPD and other inflammatory airway diseases. Basic Clin Pharmacol Toxicol. 2014;114(5):365-376. doi:10.1111/bcpt.12209

70. Beute J, Lukkes M, Koekoek EP, et al. A pathophysiological role of PDE3 in allergic airway inflammation. JCI Insight. 2018;3(2): e94888. doi:10.1172/jci.insight.94888

71. BinMahfouz H, Borthakur B, Yan D, George T, Giembycz MA, Newton R. Superiority of combined phosphodiesterase PDE3/ PDE4 inhibition over PDE4 inhibition alone on glucocorticoidand long-acting $\beta_{2}$-adrenoceptor agonist-induced gene expression in human airway epithelial cells. Mol Pharmacol. 2015;87 (1):64-76. doi:10.1124/mol.114.093393

72. Cazzola M, Calzetta L, Rogliani P, Matera MG. Ensifentrine (RPL554): an investigational PDE3/4 inhibitor for the treatment of COPD. Expert Opin Investig Drugs. 2019;28(10):827-833. doi:10.1080/13543784.2019.1661990

73. Calzetta L, Page CP, Spina D, et al. Effect of the mixed phosphodiesterase $3 / 4$ inhibitor RPL554 on human isolated bronchial smooth muscle tone. J Pharmacol Exp Ther. 2013;346(3):41 4-423. doi:10.1124/jpet.113.204644

74. Calzetta L, Cazzola M, Page CP, Rogliani P, Facciolo F, Matera MG. Pharmacological characterization of the interaction between the dual phosphodiesterase (PDE) 3/4 inhibitor RPL554 and glycopyrronium on human isolated bronchi and small airways. Pulm Pharmacol Ther. 2015;32:15-23. doi:10.1016/j. pupt.2015.03.007

75. Franciosi LG, Diamant Z, Banner KH, et al. Efficacy and safety of RPL554, a dual PDE3 and PDE4 inhibitor, in healthy volunteers and in patients with asthma or chronic obstructive pulmonary disease: findings from four clinical trials. Lancet Respir Med. 2013;1(9):714-727. doi:10.1016/S2213-2600(13)70187-5

76. Bjermer L, Abbott-Banner K, Newman K. Efficacy and safety of a first-in-class inhaled PDE3/4 inhibitor (ensifentrine) vs salbutamol in asthma. Pulm Pharmacol Ther. 2019;58:101814. doi:10.10 16/j.pupt.2019.101814 
77. Shih $\mathrm{CH}$, Wang WH, Chen CM, Ko WC. Hesperetin-5,7,3'$O$-trimethylether dually inhibits phosphodiesterase $3 / 4$ and methacholine-induced airway hyperresponsiveness in sensitized and challenged mice. Drug Des Devel Ther. 2020;14:519-526. doi:10.2147/DDDT.S227432

78. Mokry J, Urbanova A, Medvedova I, et al. Effects of tadalafil (PDE5 inhibitor) and roflumilast (PDE4 inhibitor) on airway reactivity and markers of inflammation in ovalbumin-induced airway hyperresponsiveness in guinea pigs. J Physiol Pharmacol. 2017;68 (5):721-730.

79. Blanco I, Santos S, Gea J, et al. Sildenafil to improve respiratory rehabilitation outcomes in COPD: a controlled trial. Eur Respir J. 2013;42(4):982-992. doi:10.1183/09031936.00176312

80. Smith SJ, Cieslinski LB, Newton R, et al. Discovery of BRL 50481 [3-(N,N-dimethylsulfonamido)-4-methyl-nitrobenzene], a selective inhibitor of phosphodiesterase 7: in vitro studies in human monocytes, lung macrophages, and CD8+ T-lymphocytes. Mol Pharmacol. 2004;66(6):1679-1689. doi:10.1124/mol.104.002246

81. Fortin M, D'Anjou H, Higgins ME, et al. A multi-target antisense approach against PDE4 and PDE7 reduces smoke-induced lung inflammation in mice. Respir Res. 2009;10(1):39. doi:10.1186/ 1465-9921-10-39

82. Mokry J, Joskova M, Mokra D, Christensen I, Nosalova G. Effects of selective inhibition of PDE4 and PDE7 on airway reactivity and cough in healthy and ovalbumin-sensitized guinea pigs. Adv Exp Med Biol. 2013;756:57-64.

83. Yamamoto S, Sugahara S, Ikeda K, Shimizu Y. Pharmacological profile of a novel phosphodiesterase 7A and -4 dual inhibitor, YM393059, on acute and chronic inflammation models. Eur J Pharmacol. 2006;550(1-3):166-172. doi:10.1016/j.ejphar.2006.08.023

84. Jankowska A, Swierczek A, Chlon-Rzepa G, Pawlowski M, Wyska E. PDE7-selective and dual inhibitors: advances in chemical and biological research. Curr Med Chem. 2017;24 (7):673-700. doi:10.2174/0929867324666170116125159

85. Chłoń-Rzepa G, Jankowska A, Ślusarczyk M, et al. Novel butanehydrazide derivatives of purine-2,6-dione as dual PDE4/7 inhibitors with potential anti-inflammatory activity: design, synthesis and biological evaluation. Eur J Med Chem. 2018;146:381-394. doi:10.1016/j.ejmech.2018.01.068

86. Chłoń-Rzepa G, Ślusarczyk M, Jankowska A, et al. Novel amide derivatives of 1,3-dimethyl-2,6-dioxopurin-7-yl-alkylcarboxylic acids as multifunctional TRPA1 antagonists and PDE4/7 inhibitors: a new approach for the treatment of pain. Eur J Med Chem. 2018;158:517-533. doi:10.1016/j.ejmech.2018.09.021

87. Sharma H, Lather V, Grewal AS, Pandita D. Anti-inflammatory activity and docking studies of some newer 1,3-thiazolidine2,4-dione derivatives as dual inhibitors of PDE4 and PDE7. Curr Comput Aided Drug Des. 2019;15(3):225-234. doi:10.21 74/1573409914666181003151528

88. Bender AT, Beavo JA. Cyclic nucleotide phosphodiesterases: molecular regulation to clinical use. Pharmacol Rev. 2006;58 (3):488-520. doi:10.1124/pr.58.3.5

89. Rybalkin SD, Bornfeldt KE, Sonnenburg WK, et al. Calmodulinstimulated cyclic nucleotide phosphodiesterase (PDE1C) is induced in human arterial smooth muscle cells of the synthetic, proliferative phenotype. J Clin Invest. 1997;100(10):2611-2621. doi:10.1172/JCI119805

90. Bergeron C, Tulic MK, Hamid Q. Airway remodelling in asthma: from benchside to clinical practice. Can Respir J. 2010;17(4): e85-e93. doi:10.1155/2010/318029

91. Myou S, Fujimura M, Kurashima K, Tachibana H, Hirose T, Nakao S. Effect of aerosolized administration of KF19514, a phosphodiesterase 4 inhibitor, on bronchial hyperresponsiveness and airway inflammation induced by antigen inhalation in guinea-pigs. Clin Exp Allergy. 2000;30(5):713-718. doi:10.1046/ j.1365-2222.2000.00782.x
92. Kita T, Fujimura M, Myou S, Watanabe K, Waseda Y, Nakao S Effects of KF19514, a phosphodiesterase 4 and 1 Inhibitor, on bronchial inflammation and remodeling in a murine model of chronic asthma. Allergol Int. 2009;58(2):267-275. doi:10.2332/ allergolint.08-OA-0053

93. Martinez A, Gil C. cAMP-specific phosphodiesterase inhibitors: promising drugs for inflammatory and neurological diseases. Expert Opin Ther Pat. 2014;24(12):1311-1321. doi:10.1517/ 13543776.2014.968127

94. Crimi N, Palermo F, Oliveri R, et al. Enhancing effect of dipyridamole inhalation on adenosine-induced bronchospasm in asthmatic patients. Allergy. 1988;43(3):179-183. doi:10.1111/ j.1398-9995.1988.tb00416.x

95. Oldenburger A, Maarsingh H, Schmidt M. Multiple facets of cAMP signalling and physiological impact: cAMP compartmentalization in the lung. Pharmaceuticals (Basel). 2012;5 (12):1291-1331. doi:10.3390/ph5121291

96. Wójcik-Pszczoła K, Chłoń-Rzepa G, Jankowska A, et al. A novel, pan-PDE inhibitor exerts anti-fibrotic effects in human lung fibroblasts via inhibition of TGF- $\beta$ signaling and activation of cAMP/PKA signaling. Int J Mol Sci. 2020;21(11):4008. doi:10.3390/ijms21114008

97. Giembycz MA, Maurice DH. Cyclic nucleotide-based therapeutics for chronic obstructive pulmonary disease. Curr Opin Pharmacol. 2014;16:89-107. doi:10.1016/j.coph.2014.04.001

98. Tannheimer SL, Sorensen EA, Haran AC, Mansfield CN, Wright CD, Salmon M. Additive anti-inflammatory effects of beta 2 adrenoceptor agonists or glucocorticosteroid with roflumilast in human peripheral blood mononuclear cells. Pulm Pharmacol Ther. 2012;25(2):178-184. doi:10.1016/j.pupt.2012.01.003

99. Joshi T, Yan D, Hamed O, et al. GS-5759, a bifunctional $\beta_{2^{-}}$ adrenoceptor agonist and phosphodiesterase 4 Inhibitor for chronic obstructive pulmonary disease with a unique mode of action: effects on gene expression in human airway epithelial cells. J Pharmacol Exp Ther. 2017;360(2):324-340. doi:10.11 24/jpet.116.237743

100. Tannheimer SL, Sorensen EA, Cui ZH, et al. The in vitro pharmacology of GS-5759, a novel bifunctional phosphodiesterase 4 inhibitor and long acting $\beta_{2}$-adrenoceptor agonist. J Pharmacol Exp Ther. 2014;349(1):85-93. doi:10.1124/jpet.113.210997

101. Salmon M, Tannheimer SL, Gentzler TT, et al. The in vivo efficacy and side effect pharmacology of GS-5759, a novel bifunctional phosphodiesterase 4 inhibitor and long-acting $\beta_{2^{-}}$ adrenoceptor agonist in preclinical animal species. Pharmacol Res Perspect. 2014;2(4):e00046. doi:10.1002/prp2.46

102. Liu A, Huang L, Wang Z, et al. Hybrids consisting of the pharmacophores of salmeterol and roflumilast or phthalazinone: dual $\beta_{2}$-adrenoceptor agonists-PDE4 inhibitors for the treatment of COPD. Bioorg Med Chem Lett. 2013;23(5):1548-1552. doi:10. 1016/j.bmcl.2012.11.058

103. Huang L, Shan W, Zhou Q, Xie J, Lai K, Li X. Design, synthesis and evaluation of dual pharmacology $\beta_{2}$-adrenoceptor agonists and PDE4 inhibitors. Bioorg Med Chem Lett. 2014;24(1):24 9-253. doi:10.1016/j.bmcl.2013.11.028

104. Provins L, Christophe B, Danhaive P, et al. First dual M3 antagonists-PDE4 inhibitors: synthesis and SAR of 4,6-diaminopyrimidine derivatives. Bioorg Med Chem Lett. 2006;16(7):1834-1839. doi:10.1016/j.bmcl.2006.01.006

105. Provins L, Christophe B, Danhaive P, et al. Dual M3 antagonists-PDE4 inhibitors. Part 2: synthesis and SAR of 3-substituted azetidinyl derivatives. Bioorg Med Chem Lett. 2007;17(11):3077-3080. doi:10.1016/j.bmcl.2007.03.047

106. Cazzola M, Matera MG. Emerging inhaled bronchodilators: an update. Eur Respir J. 2009;34(3):757-769. doi:10.1183/090319 36.00013109

107. Phillips G, Salmon M. Bifunctional compounds for the treatment of COPD. Annu Rep Med Chem. 2012;47:209-222. 
108. Belgrave DC, Custovic A, Simpson A. Characterizing wheeze phenotypes to identify endotypes of childhood asthma, and the implications for future management. Expert Rev Clin Immunol. 2013;9(10):921-936. doi:10.1586/1744666X.2013.836450

109. Agache I, Akdis CA. Precision medicine and phenotypes, endotypes, genotypes, regiotypes, and theratypes of allergic diseases. J Clin Invest. 2019;129(4):1493-1503. doi:10.1172/JCI124611

110. Agusti A, Bel E, Thomas M, et al. Treatable traits: toward precision medicine of chronic airway diseases. Eur Respir J. 2016;47 (2):410-419. doi:10.1183/13993003.01359-2015

111. Simpson AJ, Hekking PP, Shaw DE, et al. Treatable traits in the European U-BIOPRED adult asthma cohorts. Allergy. 2019;74 (2):406-411. doi:10.1111/all.13629
112. Rhee CK, Kim JH, Kim SY, et al. Effect of roflumilast on neutrophilic asthma model (abstract). Eur Respir J. 2017;50: PA1147.

113. Bardin P, Kanniess F, Gauvreau G, Bredenbröker D, Rabe KF. Roflumilast for asthma: efficacy findings in mechanism of action studies. Pulm Pharmacol Ther. 2015;35(Suppl):S4-S10. doi:10. 1016/j.pupt.2015.08.006

114. Fahy JV. Type 2 inflammation in asthma - present in most, absent in many. Nat Rev Immunol. 2015;15(1):57-65. doi:10.1038/nri 3786

\section{Publish your work in this journal}

The Journal of Experimental Pharmacology is an international, peerreviewed, open access journal publishing original research, reports, reviews and commentaries on all areas of laboratory and experimental pharmacology. The manuscript management system is completely online and includes a very quick and fair peer-review system. Visit http://www.dovepress.com/testimonials.php to read real quotes from published authors. 